\title{
Light Polarization-Assisted Sensing with Tilted Fiber Bragg Gratings
}

\author{
Christophe Caucheteur ${ }^{*}$, Valérie Voisin and Patrice Mégret
}

\author{
Electromagnetism and Telecommunication Department, Faculty of Engineering, University of Mons, Boulevard Dolez \\ 31, 7000 Mons, Belgium
}

\begin{abstract}
Tilted fiber Bragg gratings (TFBGs) are short-period gratings for which the refractive index modulation of the fiber core is angled by a few degrees with respect to the perpendicular to the propagation axis. They induce two kinds of light coupling: the self-backward coupling of the core mode at the Bragg wavelength and the backward coupling between the core mode and several tens of cladding modes, each with its own effective refractive index and mode field shape. The information contained in their spectral content is such that they inherently provide novel sensing modalities and improve the sensitivity in comparison to uniform fiber Bragg gratings. In particular, TFBGs are intrinsically temperatureinsensitive by referencing spectral measurements to the wavelength and power level of the core mode resonance. As the tilt angle breaks the circular symmetry of the optical fiber cross-section, TFBGs are strongly dependent on light polarization effects, which can be advantageously used when they are coated with nanoscale metal coatings or thin films. In this review, we report the latest achievements obtained with bare and coated TFBGs used for high-resolution refractometry and biochemical sensing through surface Plasmon wave generation.
\end{abstract}

Keywords: Optical fiber, sensors, plasmonics, Bragg gratings, refractometry, coatings, thin films.

\section{INTRODUCTION}

Fiber Bragg gratings (FBGs) photowritten in the core of an optical fiber are widely used as mechanical and physical sensors. They correspond to a core refractive index modulation (period $\sim 500 \mathrm{~nm}$ ) perpendicular to the propagation axis and act as bandpass filters in reflection. They are inherently sensitive to ambient temperature changes and mechanical strain but suffer from a cross-sensitivity issue when temperature and strain appear simultaneously [1]. A variant to the FBG concept, namely tilted fiber Bragg gratings (TFBGs), solves the cross-sensitivity issue while opening up the sensing modalities. This concept was explored almost as soon as FBGs were achieved but it has only found widespread applications in sensing since the last decade [2].

With a refractive index modulation angled by a few degrees with respect to the perpendicular to the propagation axis, TFBGs couple light from the fiber core to the cladding in a privileged direction, close to the perpendicular to the grating fringes. Actually, two kinds of couplings coexist: 1) the self-backward coupling of the core mode at the Bragg wavelength and 2) discrete backward couplings between the core mode and several tens of cladding modes [2]. As a result, the amplitude spectrum of TFBGs behaves like a spectral comb for which resonant wavelengths present a differential sensitivity to external perturbations. This has numerous benefits. While the core mode (Bragg) resonance is only sensitive to axial strain and temperature, the cladding mode resonances also vary with other external perturbations such

\footnotetext{
*Address correspondence to this author at the Electromagnetism and Telecommunication Department, Faculty of Engineering, University of Mons, Boulevard Dolez 31, 7000 Mons, Belgium; Tel: +32-65-374149; Fax: +32-65-374199;

Email: christophe.caucheteur@umons.ac.be
}

as bending or surrounding refractive index changes. It has been experimentally observed that the temperature dependence of cladding modes is similar to that of the core mode [3]. Hence, the effect of temperature can be straightforwardly removed from a selected cladding mode resonance by monitoring its wavelength spacing from the core mode resonance. Similarly, unwanted power fluctuations can be avoided by referencing the actual peak power to the power level of the Bragg resonance. Also, cladding mode resonances are intrinsically sensitive to surrounding refractive index changes and bending, which multiplies the sensing modalities compared to uniform FBGs. TFBGs have been demonstrated to be accurate refractometers [4-6], bending sensor $[7,8]$ and vibration sensors $[9,10]$. A third benefit of using TFBGs is that the tilt angle breaks the cylindrical symmetry of the coupling process relative to the state of polarization (SOP) of the guided core light. This brings convenient polarization-dependent light coupling mechanisms in thin film-coated TFBGs. In particular, a resonant coupling to a Plasmon wave can be achieved. When TFBGs are covered by a nanoscale noble metal layer, the cladding modes whose effective refractive index and polarization state are equal or close to those of a Plasmon wave transfer energy to it across the metallic surface. An unambiguous SPR signature appears in the TFBG amplitude spectrum, as reported in $[11,12]$. It is strongly sensitive to surrounding refractive index (SRI) changes [12-15], which yields an implementation of SPRbased (bio-)sensors easy to fabricate and to use.

The physical properties and practical applications of TFBG sensors have been recently reviewed extensively [16]. To complement this review, we present here the latest achievements obtained with thin film-coated and gold-coated TFBGs used for high-resolution refractometry and biochemical sensing. We demonstrate how the fine-tuning of 
the input SOP of the light improves the SRI sensitivity. We also report the different strategies that can be used to correlate the spectral content with the SRI value.

In the remaining of the paper, we first summarize the main features of TFBGs. We then focus on their use as refractometers. We describe the main results obtained with bare and coated TFBGs, while emphasizing on the crucial role of the light polarization on the demodulation process used to correlate the spectral content evolution with the SRI value.

\section{TFBGS CHARACTERISTICS}

As sketched in (Fig. 1a), a uniform FBG is a periodic and permanent refractive index modulation of the fiber core imprinted perpendicularly to the propagation axis $[1,2]$. It reflects a narrow spectral band centered on the so-called Bragg wavelength $\lambda_{\text {Bragg }}=2 n_{\text {eff,core }} \Lambda$ where $n_{\text {eff,core }}$ is the effective refractive index of the core mode (close to 1.45) and $\Lambda$ is the grating periodicity. The Bragg wavelength is inherently sensitive to mechanical strain and temperature, through a change of both $n_{\text {eff }}$ and $\Lambda$ [1]. However, it is totally insensitive to SRI changes since the penetration depth of the core-guided light into the cladding does not exceed a few micrometers. In practice, the optical cladding has to be partly removed or etched to expose the evanescent wave of the core mode to the surrounding medium, as reported in [17]. Using radiative gratings that couple light from the core to the cladding presents the considerable advantage to preserve the optical fiber integrity. In this context, TFBGs are particularly interesting to use.

A TFBG corresponds to a refractive index modulation angled by a few degrees relative to the perpendicular to the propagation axis (Fig. 1b). In addition to the self-backward coupling of the core mode at the Bragg wavelength, TFBG redirects some light to the cladding whose diameter is so large that several tens of cladding modes can propagate, each with its own effective refractive index $n_{\text {eff,clad. The phase }}$ matching condition for the $i^{\text {th }}$ cladding mode resonance is given by $\lambda_{\text {clad, } i}=\left(n_{\text {eff,core }}+n_{\text {eff,clad, } i}\right) \Lambda$.
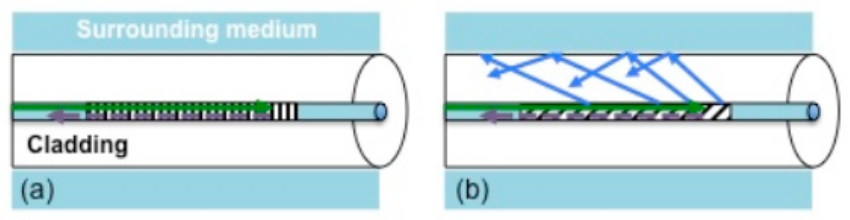

Fig. (1). Sketch of the light coupling mechanism for uniform FBGs (a) and tilted FBGs (b).

Fig. (2a) depicts the typical transmitted spectrum of a 1 $\mathrm{cm}$ long uniform FBG. It is composed of a single rejection band centered on the Bragg wavelength. Fig. (2b) depicts a typical TFBG transmission spectrum where each resonance corresponds to the coupling from the core mode to a group of backward-going cladding modes. The spectral position of a cladding mode resonance is related to its effective refractive index, which in turn depends on the optical properties of the medium surrounding the optical fiber outer surface. For instance, the cladding mode resonance close to $1540 \mathrm{~nm}$ is characterized by an effective refractive index value near
1.315 , which is the refractive index of water at this wavelength. In water, cladding mode resonances at shorter wavelengths are therefore drastically attenuated since the modes are no longer guided by the cladding-water interface. Oppositely, resonances just above $1540 \mathrm{~nm}$ remain guided. Their evanescent field penetrates the surrounding medium over distances corresponding to several wavelengths. In the jargon, the wavelength at which a mode is no longer guided is the cut-off wavelength. In practice, resonances just above this transition point are the most sensitive to SRI changes [6]. They shift towards longer (shorter) wavelengths as the SRI increases (decreases). For resonances at longer wavelengths, the modes are increasingly confined into the cladding and their SRI sensitivity decreases accordingly, reaching complete insensitivity for the core mode resonance at the Bragg wavelength. It is worth mentioning that the amplitude spectra presented in (Fig. 2) were recorded without polarization control of the input light. Also, all experimental results reported hereafter are obtained with standard single-mode optical fibers that are hydrogen-loaded to enhance their photosensitivity.

In addition to the wavelength shift, SRI changes also modify the amplitude of a cladding mode resonance, which depends on the distribution and polarization of the electromagnetic field of the mode over the fiber cross-section. When the SRI increases, the mode field becomes less confined by the cladding and extends deeper outside of the fiber. As a result, there is a decrease of the coupling strength, which in turn decreases the cladding mode resonance amplitude. We will show in the following how both the wavelength shift and the amplitude change of a given cladding mode resonance can be used for high resolution SRI sensing.

TFBGs can be manufactured in the same way as uniform ones using the transverse holographic method or the phase mask technique. We use a continuous-wave frequencydoubled Argon laser emitting at $244 \mathrm{~nm}$ and phase masks placed on a goniometer so that they can be tilted in the plane perpendicular to the writing laser beam. We use external tilt angles ranging between 5 and $10^{\circ}$ to guarantee strong coupling to cladding mode resonances with effective refractive index close to water, i.e. 1.315. For details on the manufacturing process of TFBGs and the influence of the tilt angle on the cladding mode couplings, the interested reader is invited to consult ref. [16].

\section{REFRACTOMETRIC SENSING WITH TFBGS}

\subsection{Bare TFBGs}

First experiments that demonstrate the possibility to sense SRI with TFBGs date back to 2001 [4]. Fig. (3) presents the transmitted spectral evolution of a $1 \mathrm{~cm}$ long $4^{\circ}$ TFBG immersed in calibrated oils whose refractive indices are known with an accuracy better than $210^{-4}$. The transmitted spectra measured with different SRI values are drawn on the same graph with a vertical offset. The values of the corresponding refractive index are written next to the curves. As expected, the amplitudes of the high order cladding mode resonances progressively decrease when the SRI increases. When the SRI reaches $n_{\text {eff,clad,i }}$, the coupling strength of the $i^{\text {th }}$ cladding mode decreases. When the SRI matches $n_{\text {eff,clad,i }}$, the $i^{\text {th }}$ cladding mode is no longer guided. Spectrally, this 

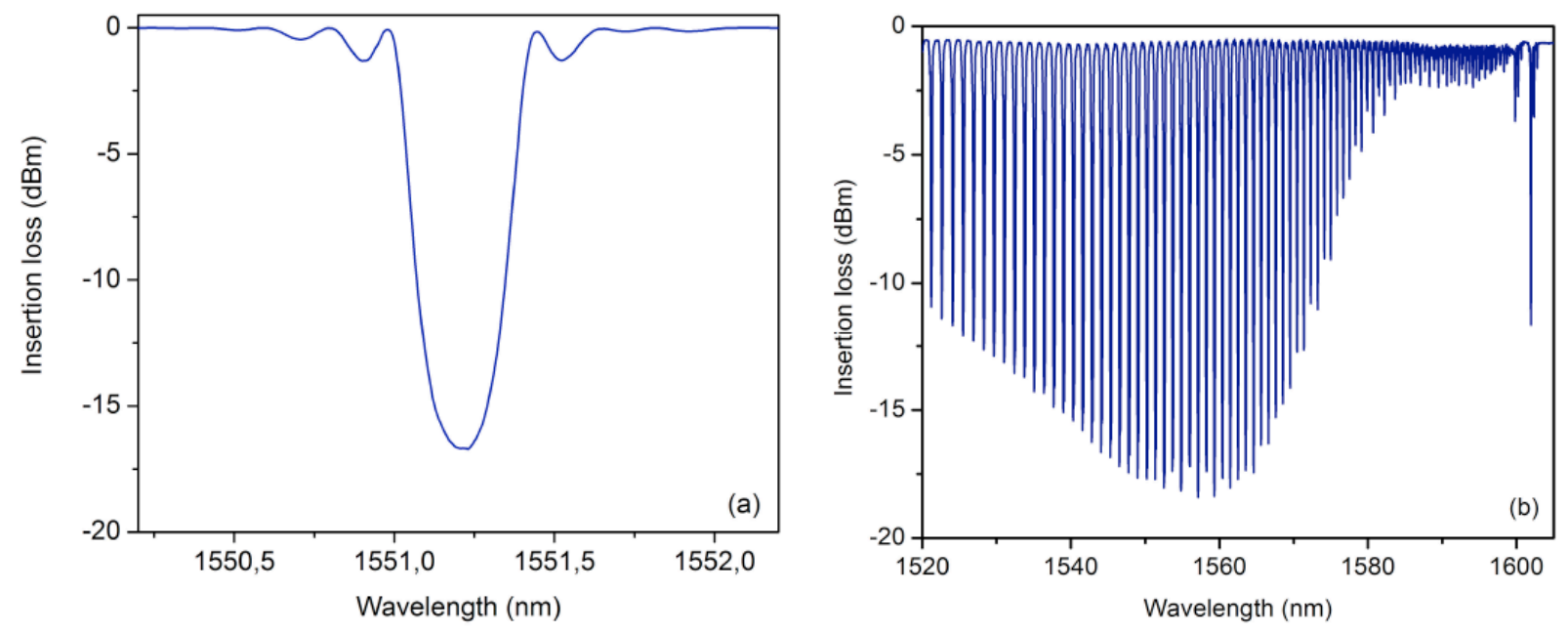

Fig. (2). Typical transmission spectrum of a $1 \mathrm{~cm}$ long uniform $\mathrm{FBG}$ and a $10^{\circ} \mathrm{TFBG}$ measured in air.

behavior is marked by a smoothing of the cladding modes around their respective coupling wavelengths and corresponds to an increase of the radiation coupling.

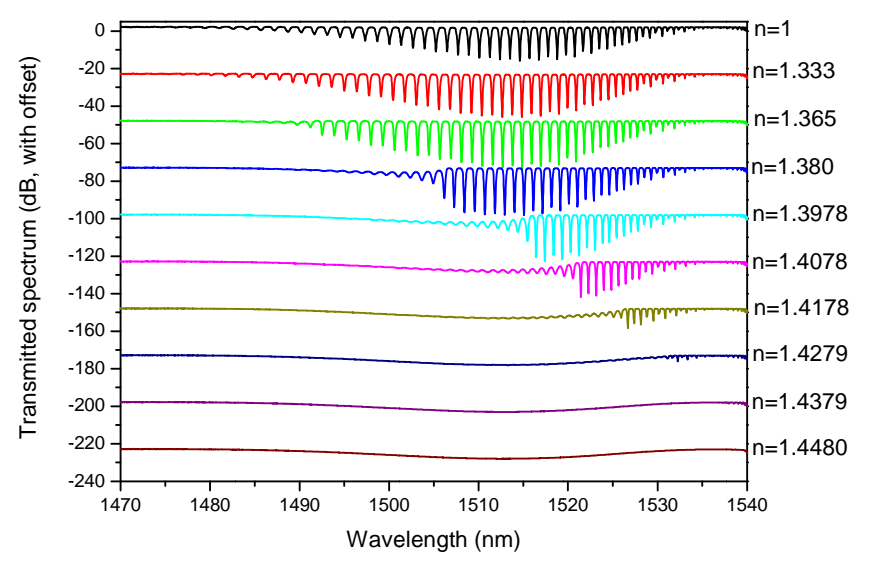

Fig. (3). Evolution of a $4^{\circ}$ TFBG transmitted spectrum as a function of the SRI (vertical axis with an offset).

Several data processing techniques were reported to quantitatively correlate the spectral content with the SRI value, either through the exploitation of a global spectral evolution or a local spectral feature change. The first method relies on the monitoring of the area delimited by the cladding mode resonance spectrum, via a computation of the upper and lower envelopes. For increasing SRI values, the area is decreasing, as resonances gradually disappear when the SRI reaches the cut-off points of each cladding mode [4,5]. In practice, the normalized area is used, i.e. the area computed for a given SRI is divided by the one measured for the same grating immersed in a reference medium (air or water). Another technique tracks the wavelength shift and amplitude variation of individual cladding mode resonances as they reach the cut-off [6], therefore presenting the maximum sensitivity to the SRI. The use of the polarization dependent loss (PDL) associated to the transmitted spectrum of TFBG has also been reported as a demodulation technique for TFBG refractometers [18]. The use of an optical time domain re- flectometer (OTDR) has been proposed as well to interrogate a cascade of identical TFBGs that can be selectively resolved in the time domain by measuring their insertion loss on the OTDR trace [19].

All these techniques present minimum detectable SRI changes of $\sim 10^{-4}$ RIU, just because bare TFBGs in standard single-mode optical fiber cannot inherently sense smaller changes. In terms of wavelength tracking, this result is obtained with a sensitivity that peaks between 10 and 15 $\mathrm{nm} / \mathrm{RIU}$ for the modes near cut-off. The Bragg wavelength always provides an absolute power and wavelength reference, which can be used to remove uncertainties due to light power fluctuations or temperature changes. It was indeed demonstrated that all resonances - including the Bragg one present the same temperature sensitivity [20]. TFBGs thus allow temperature-insensitive SRI measurements.

However, for high-resolution SRI sensing including biochemical measurements, it is necessary to decrease the limit of detection down to a level of $10^{-5}$ RIU at least [21]. The association of TFBGs with functional coatings is a convenient way to reach this purpose. In the following, we review the different strategies that were successfully applied during the past few years. The ultimate solution consists in using a nanoscale gold coating that allows surface Plasmon wave generation and remains the best overlay to conduct biochemistry experiments.

\subsection{Syndiotactic Polystyrene-Coated TFBGs}

The dip-coating technique was exploited to deposit thin films of syndiotactic polystyrene onto TFBGs, which was chosen for its refractive index slightly higher than the one of silica $(n=1.578)$. It mainly consists of immersing the fibersubstrate into a chloroform solution of the polymer and then of withdrawing it at a well-controlled speed. The deposition was completely automated by a controlled system, which permitted a constant withdrawal speed of $10 \mathrm{~cm} / \mathrm{min}$, ensuring the formation of a conformal coating of uniform thickness all along the grating length. Solutions at different concentration of the polymer, namely $8 \%$ and $9 \%$, were used at room temperature to obtain different overlay thicknesses of the order of several hundreds of nanometers (about $250 \mathrm{~nm}$ 
and $300 \mathrm{~nm}$, respectively). It induces a red shift of the cladding mode resonances, which depends on the coating thickness, as reported in $[22,23]$. The coating tends to increase the effective refractive index of the cladding modes. As a result, the range of sensitivity extends beyond 1.45.

Fig. (4) depicts the normalized area evolution in the SRI range $1.33-1.47$ for bare and coated TFBGs, with $8 \%$ and 9 $\%$ of polystyrene, respectively. For each configuration, the values are normalized with respect to the spectrum measured in water. The coating induces particular effects in the normalized area evolution that can be split into three different regions of interest. First, for SRI values lower than 1.38, the coated gratings exhibit a lower sensitivity to the SRI in comparison to the bare gratings. Then, for SRI values up to 1.44, the two configurations sensitivities are comparable. The evolutions of the normalized area are indeed decreasing monotonically, with similar slopes. Finally, while the standard configuration can be practically used for SRI measurements only in the range $1-1.45$ (the normalized area value tends to 0 when the SRI is equal to 1.45 ), the coated structures are able to extend the measuring range, demonstrating sensitivity also for SRI values greater than 1.47.

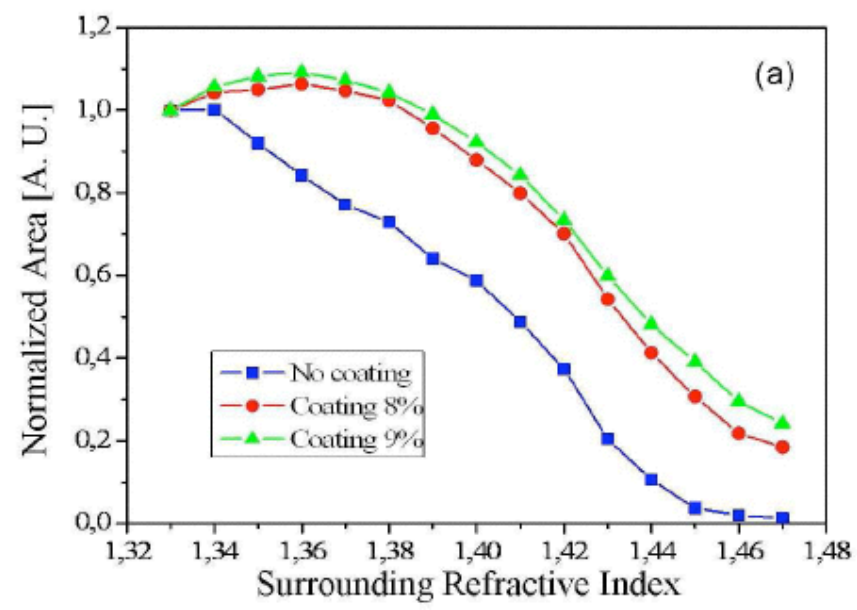

Fig. (4). Evolution of the normalized area of the transmitted spectrum versus the SRI for a $5^{\circ} \mathrm{TFBG}$.

\subsection{Transparent Thin Film-Coated TFBGs}

The high order cladding modes of standard single mode optical fiber appear in quasi-degenerate pairs corresponding to mostly radially or mostly azimuthally polarized light, socalled P-polarization and S-polarization modes [24]. It was recently demonstrated that a Zinc oxide $(\mathrm{ZnO})$ overlay characterized by a refractive index equal to 1.9 (thus well above the one of silica) considerably decreases the degeneracy between the orthogonally polarized mode families and enhances the refractometric sensitivity.

$\mathrm{ZnO}$ was deposited by a radio-frequency sputtering process, as explained in [25]. Fig. (5) compares the results obtained for a bare TFBG and a $400 \mathrm{~nm}$ coated TFBG in the wavelength range $1540 \mathrm{~nm}-1580 \mathrm{~nm}$ (the Bragg wavelength is around $1592 \mathrm{~nm}$ ). It clearly appears that the $\mathrm{ZnO}$ coating enhances the wavelength separation between the $\mathrm{S}$ and $\mathrm{P}$ orthogonal modes, compared to bare TFBGs. This behavior results from the fact that TFBGs break the optical fiber cylindrical symmetry by coupling light from the core to the cladding in a privileged direction, close to the perpendicular to the grating planes. Actually, they couple light into $\mathrm{P}\left(\mathrm{TM}_{0 \mathrm{n}}\right.$ and $\left.\mathrm{EH}_{1 \mathrm{n}}\right)$ and $\mathrm{S}\left(\mathrm{TE}_{0 \mathrm{n}}\right.$ and $\left.\mathrm{HE}_{1 \mathrm{n}}\right)$ mode families. In practice, $s$ modes are tangentially polarized at the outer surface boundary and therefore, they more difficultly penetrate it. This is not the case for $p$ modes that are radial. These ones readily localize in the coating, which increases their effective refractive index as the $\mathrm{ZnO}$ coating has a refractive index by far higher than the one of silica. As a result, $p$ modes resonances are shifted to higher wavelengths with respect to $\mathrm{s}$ modes, which is spectrally manifested by a pronounced spacing between both mode families.

Fig. (6) presents the results of numerical simulations conducted using a finite-difference complex mode solver in cylindrical coordinates (FIMMWAVE from Photon Design Inc.). A four-layer model was considered for the optical fiber: a Germanium-doped core $(8 \mu \mathrm{m})$ was surrounded by a pure silica cladding $(125 \mu \mathrm{m})$, a $\mathrm{ZnO}$ coating (its thickness was varied between 0 and $400 \mathrm{~nm}$ ) and an infinite external medium (air in this case). The $\mathrm{ZnO}$ coating was simulated as a uniform medium for which the refractive index has been set to 1.9. Simulations yield the effective refractive indices of the modes present in the considered configuration. For each mode computed by the solver, we use the TFBG phase matching condition to estimate the corresponding resonance wavelength. This is obtained by considering 1.447 for the effective refractive index of the core mode $\left(\mathrm{n}_{\text {eff,core }}\right)$ and 557 $\mathrm{nm}$ for the grating period $(\Lambda)$. The wavelength spacing is computed as the difference in wavelength between adjacent $\mathrm{p}$ and $\mathrm{s}$ modes. Fig. (6) presents the wavelength separation evolution as a function of the position of the considered resonance with respect to the core mode resonance. It confirms that a high refractive index transparent overlay drastically enhances the wavelength spacing between adjacent orthogonal polarization modes. It increases with the coating thickness and reaches more than $500 \mathrm{pm}$ for high order cladding mode resonances. Therefore, the wavelength separation is such that it allows the unambiguous selection of the $\mathrm{s}$ and $\mathrm{p}$ mode families.

Fig. (7) depicts the wavelength evolution of the cladding mode resonance located just beyond the cut-off as a function of the SRI value (total SRI change equal to $8.010^{-3}$ RIU around 1.39). The $\mathrm{s}$ and $\mathrm{p}$ modes of bare TFBGs behave equally, with a refractometric sensitivity equal to 25 $\mathrm{nm} / \mathrm{RIU}$. The $\mathrm{s}$ and $\mathrm{p}$ mode sensitivities of the $100 \mathrm{~nm}$ coated TFBG were computed equal to $75 \mathrm{~nm} / \mathrm{RIU}$ and $110 \mathrm{~nm} / \mathrm{RIU}$, respectively. For $200 \mathrm{~nm}$ coated TFBGs, the behavior becomes non linear. For slight SRI change up to $2.510^{-3}$ RIU, sensitivities were computed equal to $210 \mathrm{~nm} / \mathrm{RIU}$ and 179 $\mathrm{nm} / \mathrm{RIU}$ for the $\mathrm{s}$ and $\mathrm{p}$ modes, respectively, yielding a more than 8 times enhancement with respect to bare TFBGs. As a consequence, the limit of detection for $\mathrm{ZnO}$ coated TFBGs is of the order of $10^{-5}$ RIU.

\subsection{Gold-Coated TFBGs}

It has also been recently demonstrated that the addition of a nanoscale gold coating overlay on the TFBG outer surface considerably enhances the refractometric sensitivity through the generation of surface Plasmon resonances (SPR) $[11,12]$. 

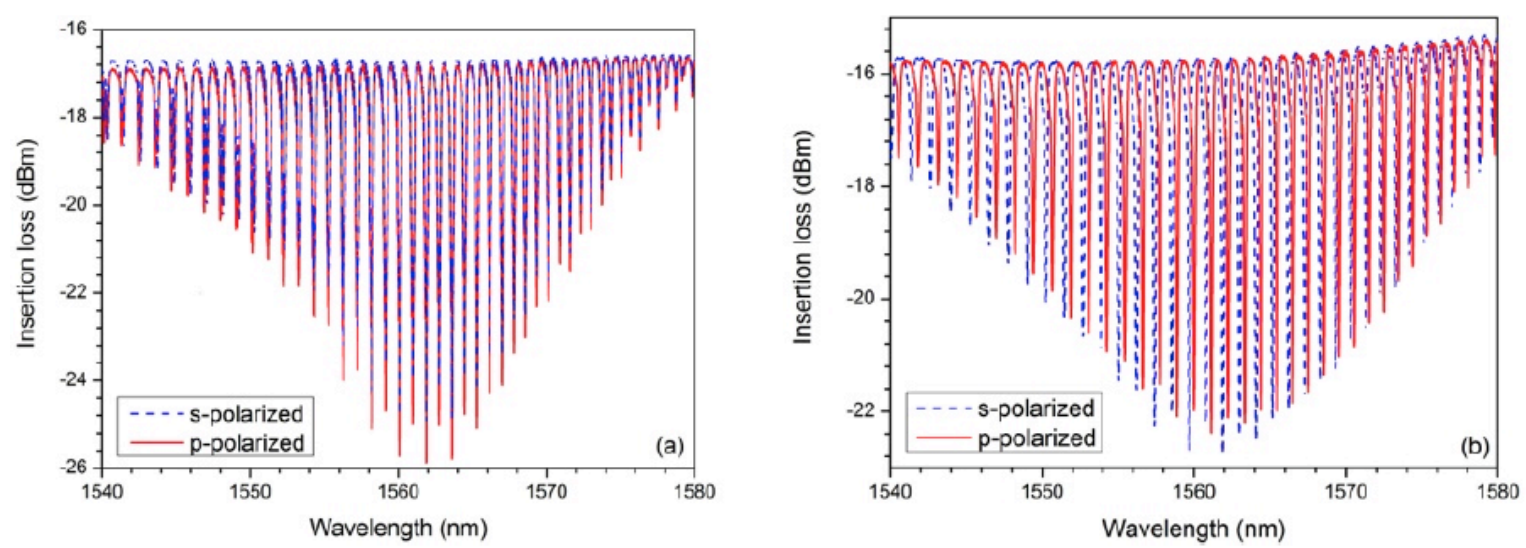

Fig. (5). Transmitted amplitude spectra of a bare TFBG (a) and a $400 \mathrm{~nm} \mathrm{ZnO}$ coated TFBG (b) in air for the s and p polarized states.

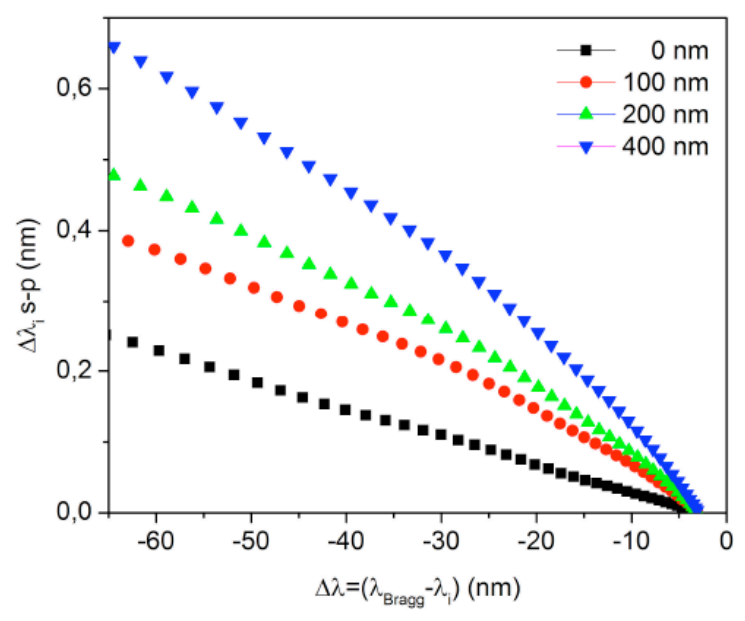

Fig. (6). Simulated wavelength spacing between $s$ and $p$ modes for three different coating thicknesses in air.

Plasmon waves result from collective excitations of electrons at the boundary between a metal and a dielectric [26,27]. In bulky configurations, measurements are most often obtained by launching light beams from a prism to a thin metallic interface at an angle such that light is totally reflected. When the component of the light propagation constant along the interface and its polarization state match those of a Plasmon excitation at the other side of the metal, light energy couples to the plasmon. (Bio-) chemical sensors are obtained by grafting receptors on the gold surface. By attracting the analytes to detect (sensing mechanism based on the antigen/antibody affinity), they affect the effective refractive index seen by the Plasmon wave, which is in turn detected through an SPR shift. The sensitivity to the SRI often ranges in the order of $10^{-6}-10^{-7}$. Various implementations of SPR sensors on optical fibers have appeared, opening the path to applications impossible with the bulky prism configuration, e.g. in situ measurements and remote monitoring. To realize optical fiber SPR sensors, light guided in the fiber core has to be brought into contact with the outer medium. Hence, the first reported configuration consisted of a multimode fiber with a section of cladding removed (or thinned) to allow interactions between the core mode and the metal coating on the fiber [28]. Interactions have been enhanced with Dshaped and side-polished single-mode fibers that expose the

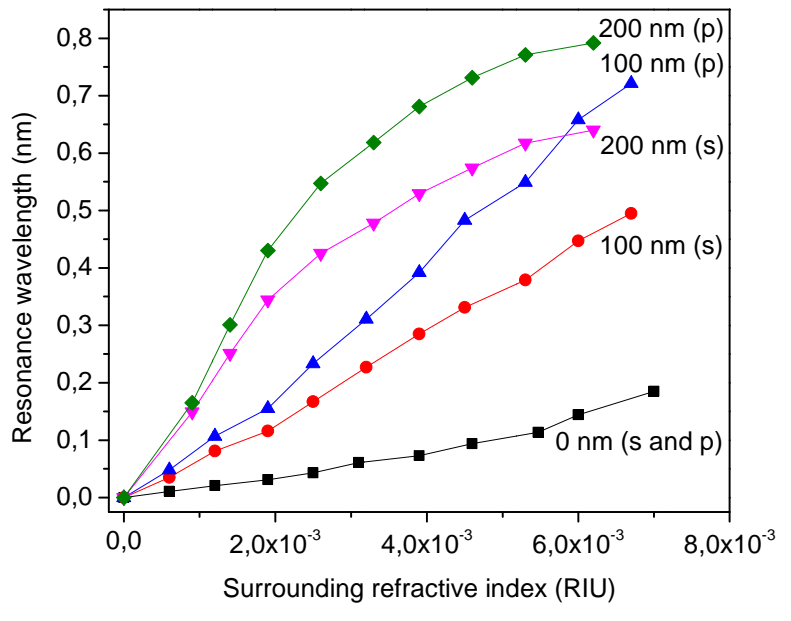

Fig. (7). Wavelength shift of the cladding mode resonance located right after the cut-off wavelength as a function of slight SRI changes.

evanescent field of the core mode to the metal coating deposited on the flat side [29]. This etching or polishing process drastically weakens the fibers and prevents their use in practical applications. At contrary, the use of radiative tilted gratings imprinted in the fiber core presents the considerable advantages of keeping the fiber integrity while yielding temperature-insensitive measurements.

The gold overlay can be successfully deposited using well-established technologies, such as electroless deposition, electroplating or sputtering. The latter technique is the most often encountered. Two consecutive depositions are made in the same conditions, with the optical fibers rotated by $180^{\circ}$ between both processes, to ensure that the whole outer surface is covered by gold. The optimum gold thickness has been practically found to range between 30 and $50 \mathrm{~nm}$. This two-step deposition results in a slightly non-uniform gold thickness, which is not at all detrimental for SPR generation. Also, as the vacuum is obtained in the chamber starting from ambient air and not from an Argon bottle, there is no need for a 2-3 nm thick adhesion layer in Chromium or Titanium between the silica surface and the gold coating.

As already noted, the tilt angle breaks the cylindrical symmetry of the coupling mechanism relative to the SOP of the input core light. P-polarized high order cladding modes 
have their electric field polarized mostly radially at the cladding-metal boundary while S-polarized cladding modes are azimuthally polarized (hence tangential to the metal surface). The transfer of energy from a cladding mode resonance to the Plasmon wave can only occur when two conditions are simultaneously fulfilled: 1) phase matching between the cladding mode and the Plasmon wave and 2) polarization of the electric field of the mode aligned perpendicularly to the coating surface (i.e. radial polarization). Hence, P-polarized input light leads to an efficient transfer of energy from a cladding mode to a surface Plasmon wave on the outer surface of the nanoscale metal layer deposited on the optical fiber outer surface. This transfer of energy is identified by a pronounced decrease of the amplitude of the outcoupled cladding mode resonance in the TFBG transmission spectrum, as reported in [11]. Oppositely, S-polarized light does not tunnel efficiently through the metal and the corresponding cladding modes remain unaffected by Plasmon wave effects. This peculiar behavior is depicted in (Fig. 8) that presents two orthogonal amplitude transmitted spectra for a gold-coated TFBG immersed in water. A pronounced amplitude decrease locally occurs for the $\mathrm{P}$ spectrum for cladding mode resonances whose effective index corresponds to the one of the Plasmon wave.

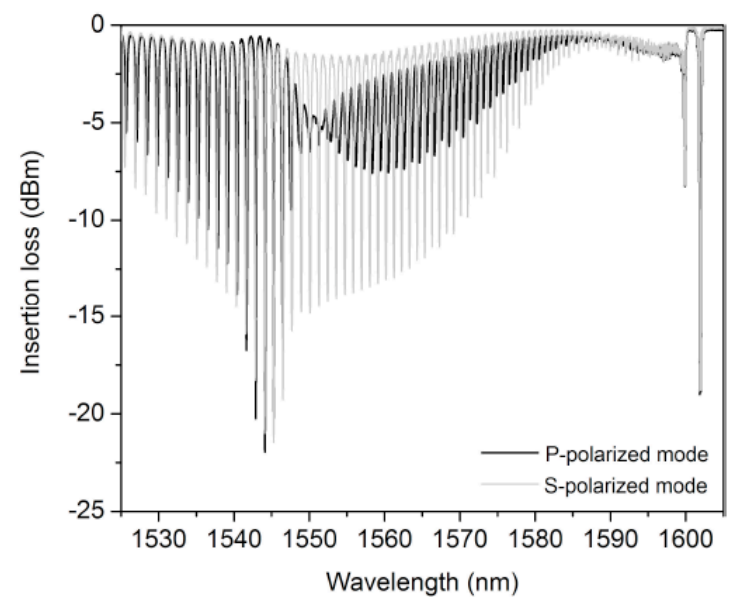

Fig. (8). Polarized transmission spectra of a gold-coated TFBG in water.

SRI sensing over a large range is possible by tracking the wavelength shift of the most attenuated cladding mode resonance, which yields SRI sensitivity of $\sim 500 \mathrm{~nm} /$ refractive index unit (RIU) in the range between 1.32 and 1.42. However, in most applications of SPR refractometry, the purpose is not absolute sensing over large SRI ranges but rather the detection of very small changes, due for instance to biomaterials attachment on a localized area of the gold surface. In this case, the most strongly coupled cladding mode resonances cannot be used for detection because they are too attenuated and broadened by the Plasmon to reveal fine spectral and amplitude shifts. In bulky configurations, this issue is addressed by a careful handling of optical and electrical noise sources and by using reference channels. In TFBGs, this is solved by monitoring the evolution of a selected resonance located just off the SPR mode [11,30]. These resonances are only partly attenuated by the SPR effect so that they keep a significant amplitude and remain narrowband.
Because of their location on the shoulder of the SPR envelope, the main effect of small spectral shifts of the SPR is to increase or decrease the resonance amplitudes depending on the direction of the SPR shift. It was also demonstrated recently that the differential behavior between two orthogonally polarized TFBG transmitted spectra can be used for demodulation purposes. We showed that narrowband resonant features (also located at wavelengths just below the SPR center) present a wavelength shift that enables refractive index measurements with a resolution better than $10^{-5}$ RIU, achieved through a quality factor of $10^{5}$ and signal-tonoise ratio greater than $50 \mathrm{~dB}[13,14]$. This particular finding was supported by a recent theoretical analysis that showed how the presence of the gold layer shields azimuthally polarized cladding modes from the outside environment while allowing radially polarized ones to tunnel through and excite Plasmons [15].

Fig. (9) presents a zoom around the SPR region of the S and $\mathrm{P}$ modes transmitted amplitude spectra. It is obvious that $\mathrm{S}$ and $\mathrm{P}$ modes come in pairs. They are labeled as a function of their position with respect to the most important one in the $\mathrm{P}$ spectrum (mode 0 in the numbering, according to [31]). This numbering reveals well the peculiar features of the TFBG spectral comb. Indeed, for short wavelengths (mode 2), the behavior is similar to that of bare TFBGs in air, with the $\mathrm{P}$ mode wavelength longer than the $\mathrm{S}$ one. Then, getting closer to the SPR mode and for each cladding mode resonances pair, the $\mathrm{P}$ mode wavelength increases less than the $\mathrm{S}$ one. The crossing point occurs for the 0 mode - so-called "apolarized" resonance - because it has the same effective refractive index for all the polarization states. This resonance is also characterized by the minimum PDL since it is defined as the absolute value of the difference between the $\mathrm{S}$ and $\mathrm{P}$ modes [13]. To the right of the 0 mode, the $P$ resonances appear on the short wavelength side of the $\mathrm{S}$ ones. The $\mathrm{P}$ mode begins to localize in the gold sheath as it approaches the SPR, which lowers its effective refractive index (due to the small value of the gold refractive index). The $\mathrm{S}$ mode is tangentially polarized at the gold boundary and hardly penetrates it.

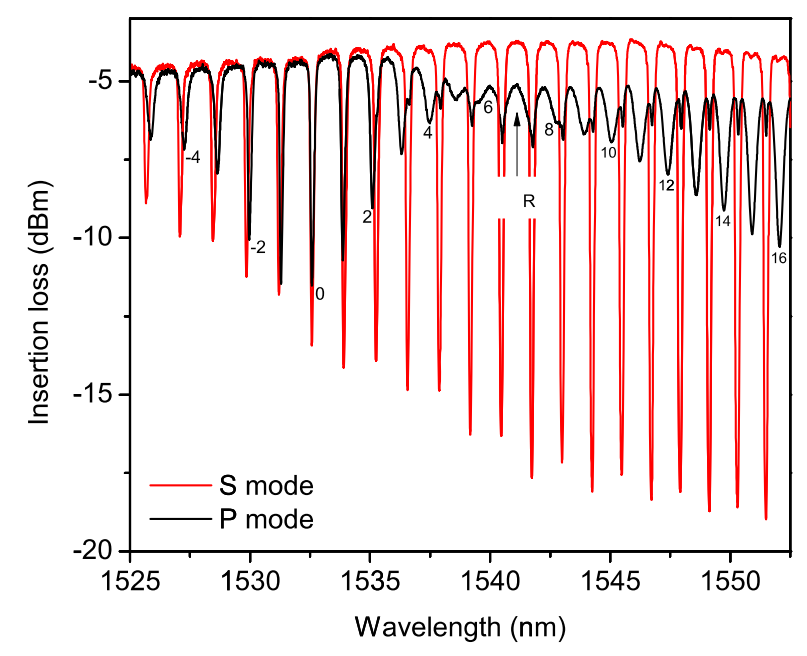

Fig. (9). Transmitted amplitude spectra for two orthogonal SOPs (S and $\mathrm{P}$ polarization modes) of a TFBG immersed in salted water $(\mathrm{SRI}=1.338)$. 
Numerical simulations can be readily conducted with Fimmwave to assess the experimental trends. Fig. (10) presents the mode loss (imaginary part of the effective refractive index) of the modes as a function of the corresponding resonance wavelength (associated with the real part of the effective refractive index) for a standard single mode fiber covered by a $30 \mathrm{~nm}$ gold layer and immersed liquid (refractive index=1.34). The SPR mode is the one for which the mode loss is the highest. In complete agreement with experimental results, $\mathrm{S}$ modes $\left(\mathrm{TE}_{0 \mathrm{n}}\right.$ and $\left.\mathrm{HE}_{1 \mathrm{n}}\right)$ farthest from the SPR center appear on the short wavelength side of their P mode adjacent resonances $\left(\mathrm{TM}_{0 \mathrm{n}}\right.$ and $\left.\mathrm{EH}_{1 \mathrm{n}}\right)$. For mode 0 , the wavelength positions of the $\mathrm{TM}_{0 \mathrm{n}}$ and $\mathrm{HE}_{1 \mathrm{n}}$ mode nearly coincide. Proceeding forward along the wavelength scale, S modes then appear on the long wavelength side of the $\mathrm{P}$ modes. The cross over point, where the $\mathrm{P}$ mode shifts to the left side of the $\mathrm{S}$ mode is highlighted in the top section of each subplot, which shows the location of the $\mathrm{S}$ resonance relative to the $\mathrm{P}$ resonance of each pair, as a function of the $\mathrm{S}$ resonance wavelength.

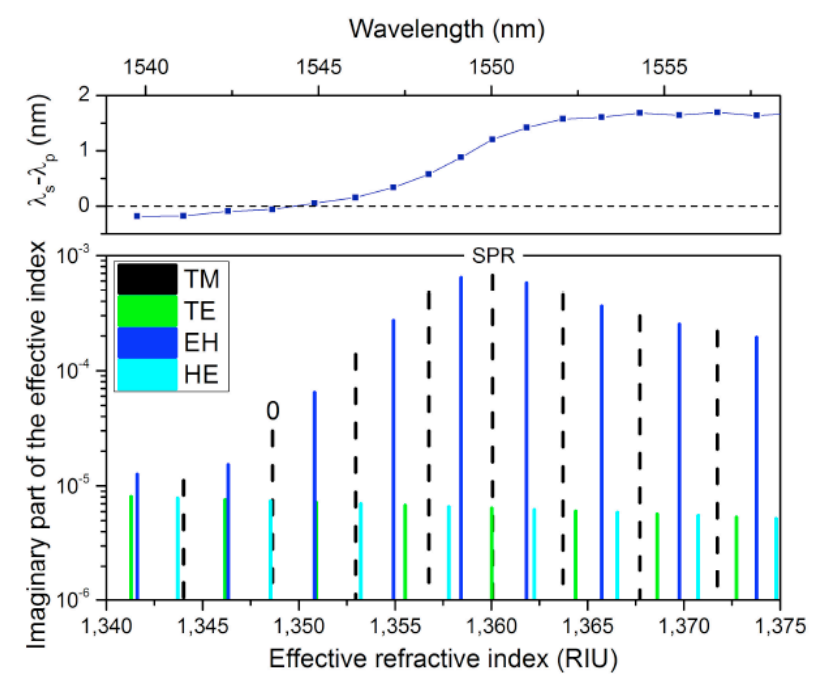

Fig. (10). Simulated mode loss as a function of the resonance wavelength for a standard single mode fiber covered by a $30 \mathrm{~nm}$ gold layer immersed in liquid of refractive index equal to 1.34.

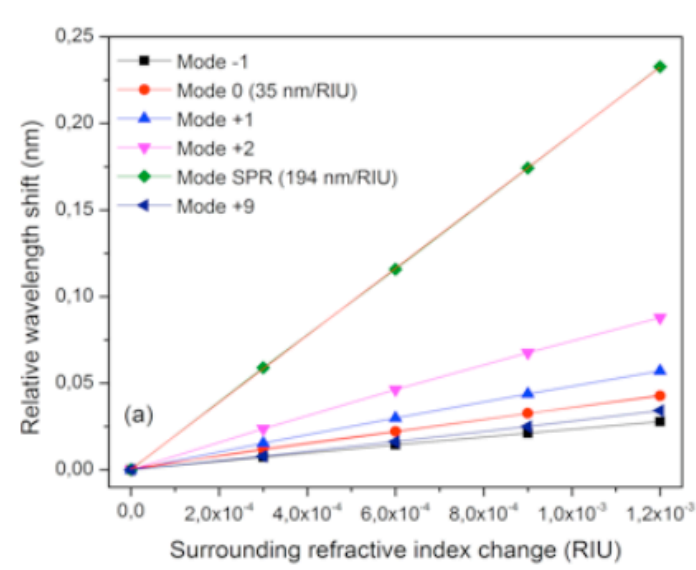

Fig. (11) depicts the relative wavelength shifts and relative mode loss variations for selected resonances when the SRI is varied in a range of $1.210^{-3} \mathrm{RIU}$. The results confirm the already established finding that the SPR mode is the most sensitive with a wavelength shift computed equal to 194 $\mathrm{nm} / \mathrm{RIU}$ [12]. However, its strong attenuation prevents its proper use in practice. Therefore, it is clear that the wavelength shift approach is inherently limited in ultimate accuracy: for SRI changes of $3.010^{-4}$ RIU, all other modes than the SPR one shift by less than $25 \mathrm{pm}$. Contrarily, amplitude changes of the order of $1 \mathrm{~dB}$ are much easier to measure, especially at absolute power levels between -10 and -25 $\mathrm{dBm}$. It is the reason why the use of low loss standard single mode fiber at near infrared wavelengths presents such a great advantage for SPR instrumentation.

Hence, in biosensing experiments relying on the use of TFBGs, the demodulation technique is based on the monitoring of the amplitude of the mode +2 , situated slightly below the SPR mode. This method has been successfully applied to perform molecular recognition with aptamers as molecular receptors [30]. Aptamers are synthetic DNA sequences that bind with high specificity to a given target. Gold-coated TFBGs have been used to monitor the functionalization of the fiber by the aptamers in real-time and to detect the presence of the aptamer's target, in various concentrations of thrombin in buffer and serum solutions. The dissociation constant has also been computed through the realization of a "Ladder" test. Plasmonic TFBG sensors were also used to detect protein down to $\mathrm{pM}$ concentrations [31].

\subsection{Nanoparticules Coated TFBGs}

Another technique has been proposed to increase the refractometric sensitivity of TFBGs. TFBGs were coated with chemically synthesized silver nanowires of $\sim 100 \mathrm{~nm}$ in diameter and a few micrometers in length. These nanowires were synthesized as described in [32]. Depositions were made by immersing TFBGs in the nanowire solution for 24 h. Fig. (12) displays the coated fiber surface measured by scanning electron microscopy (SEM).

It was then experimentally observed that the nanowire coating increases the SRI sensitivity through the excitation of localized SPR. As depicted in (Fig. 13), a 3.5-fold in-

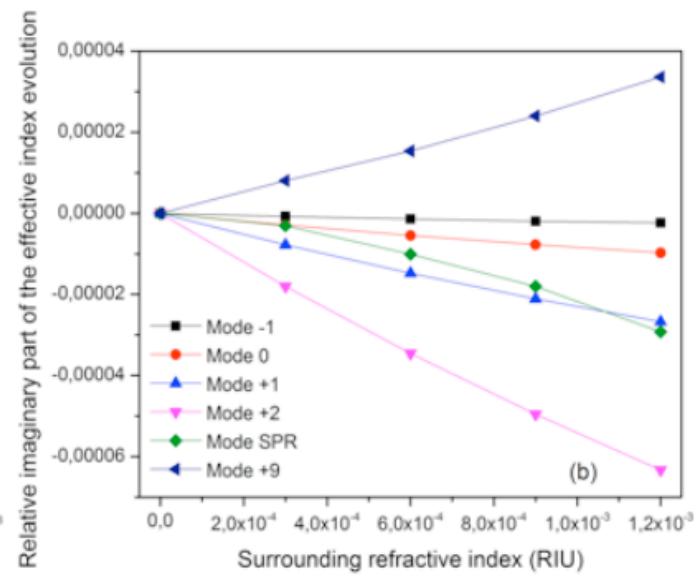

Fig. (11). Simulated relative wavelength shift (left) and relative mode loss variation (right) of selected cladding mode resonances for an SRI change of $1.210^{-3} \mathrm{RIU}$. 
crease was obtained in comparison to bare TFBGs. The linear regression yields a slope equal to $185 \mathrm{~nm} / \mathrm{RIU}$. Light polarization effects were also investigated but the conclusions were that both mode families have the same SRI sensitivity. This surely results from the fact that the coating is very dispersed, with no more than $20 \%$ of the outer surface of the optical fiber covered with nanowires.

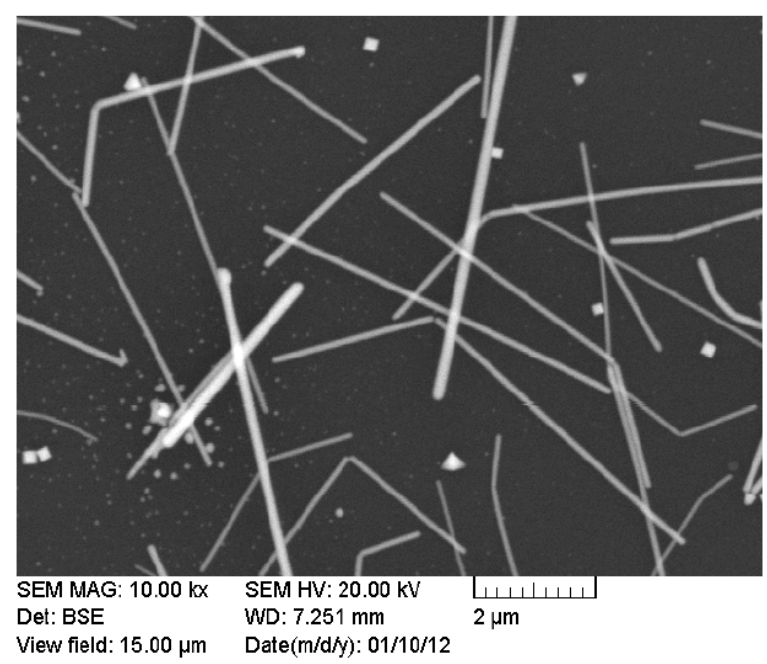

Fig. (12). SEM image of the fiber surface coated with silver nanowires [33].

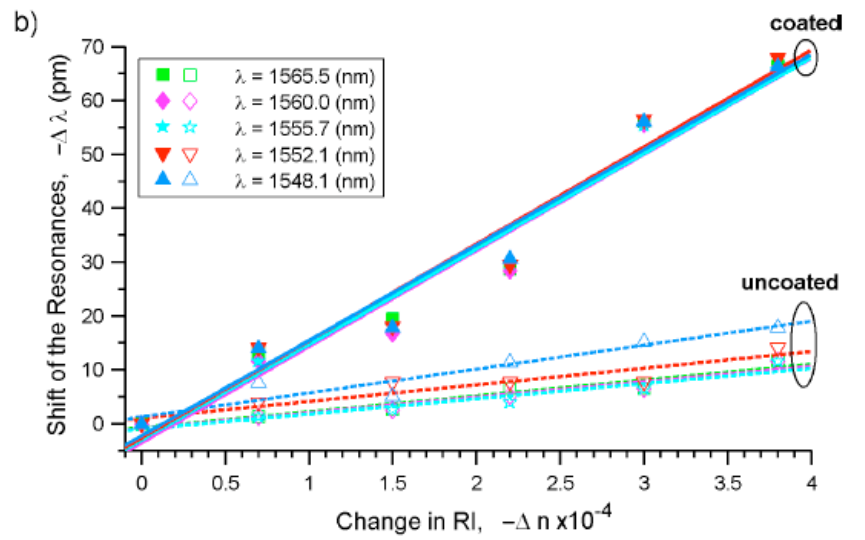

Fig. (13). Comparison between the wavelength shift of cladding mode resonance as a function of SRI changes before and after nanowires deposition [33].

Very recently, gold nanoparticles have been deposited on TFBGs using chemical vapor deposition (CVD) process [34]. Again, a differential behavior was reported between Pand S-polarized lightwaves, the former being the most attenuated in the amplitude spectrum due to an important scattering by the metal nanoparticles.

\subsection{TFBGs Sense Materials Formation onto their Sur- face}

As it acts as an evanescent wave sensor, the TFBG response can be made very sensitive to the deposition of about any kind of thin coating on the fiber outer surface. The obtained sensitivity is dependent on the overlap between the light outcoupled from the fiber core and the coating. This in turn varies with the refractive index, absorption, thickness of the coating and also depends on the medium surrounding the coating. Over the last few years, several successful demonstrations of sensitive material formation have been reported, like (1) the detection of successive depositions of individual $1.1 \mathrm{~nm}$ thick polyelectrolyte coatings [35], (2) the formation and growth of nanoparticles of copper deposited by pulsed chemical vapour deposition [36] and (3) the formation of polymer brushes using the "grafting from method" [37]. In all cases, the accuracy with which individual TFBG resonances can be followed during the deposition allows for the detailed monitoring of the processes but also to evaluate the films properties as they form.

\section{CONCLUSION}

In conclusion, TFBGs are optical fiber refractometers particularly well suited for in situ measurements in very small volumes. While bare TFBGs are only slightly sensitive to light polarization effects (with the high order cladding modes more influenced), the addition of a nanoscale layer or thin film considerably modifies the spectral behavior. Light polarization comes into play and becomes the key parameter to optimize for demodulation purposes. In most cases, a pronounced differential behavior has been reported between Spolarized and P-polarized modes, the latter being the most sensitive, as they have a radial symmetry and can therefore easily penetrate in the surrounding layer.

TFBGs present many key benefits such as easiness to fabricate, high sensitivity and self-immunity to unwanted temperature fluctuations. As they occupy a large wavelength range (several tens of nanometers), their wavelength multiplexing capability is probably their main weakness but techniques have been deployed to overcome this limitation $[19,38]$.

Studying light polarization effects in coated TFBGs is a quite new research subject, for which it remains a lot of paths to explore, not only for refractometric sensing purposes. We thus hope that this article will stimulate further research in this field.

\section{CONFLICT OF INTEREST}

The authors confirm that this article content has no conflicts of interest.

\section{ACKNOWLEDGEMENTS}

C. Caucheteur is the Research Associate of the Belgian F.R.S.-FNRS. He holds an ERC (European Research Council) Starting Independent Research Grant (Grant agreement No grant agreement $\mathrm{N}^{\circ} 280161-$ http:/hosting.umons.ac.be/erc-prosper).

\section{REFERENCES}

[1] Othonos A, Kalli K. Fiber Bragg gratings: fundamentals and applications in telecommunications and sensing. Artech House, Boston, Mass 1999.

[2] Erdogan T, Sipe JE. Tilted fiber phase gratings. J Opt Soc Am A 1996; 13: 296-313. 
[3] Chen C, Albert J. Strain-optic coefficients of the individual cladding modes of a single mode fiber: theory and experiment. Electron Lett 2006; 48: 1027-28.

[4] Laffont G, Ferdinand P. Tilted short-period fiber-Bragg-grating induced coupling to cladding modes for accurate refractometry. Meas Sci Technol 2001; 12: 765-70.

[5] Caucheteur C, Mégret P. Demodulation technique for weakly tilted fiber Bragg grating refractometer. Photon Technol Lett 2005; 17: 2703-5.

[6] Guo T, Chen C, Laronche A, Albert J. Power-referenced and temperature-calibrated optical fiber refractometer. Photon Technol Lett 2008; 20: 635-7.

[7] Baek S, Jeong Y, Lee B. Characteristics of short-period blazed fiber Bragg gratings for use as macro-bending sensors. Appl Opt 2002; 41: 631-6.

[8] Shao LY, Xiong L, Chen C, Laronche A, Albert J. Directional bend sensor based on re-grown tilted fiber Bragg grating. J Lightwave Technol 2010; 28: 2681-7.

[9] Guo T, Ivanov A, Chen C, Albert J. Temperature-independent tilted fiber grating vibration sensor based on cladding-core recoupling. Opt Lett 2008; 33: 1004-6.

[10] Guo T, Lu C, Tam HY. VCSEL-based tilted fiber grating vibration sensing system. IEEE Photon Technol Lett 2010; 22: 1235-7.

[11] Shevchenko Y, Albert J. Plasmon resonance in gold-coated tilted fiber Bragg gratings. Opt Lett 2007; 32: 211-3.

[12] Shevchenko Y, Chen C, Dakka MA, Albert J. Polarizationselective grating excitation of plasmons in cylindrical optical fibers. Opt Lett 2010; 35: 637-9.

[13] Caucheteur C, Shevchenko Y, Shao LY, Wuilpart M, Albert J. High resolution interrogation of tilted fiber grating SPR sensors from polarization properties measurement. Opt Express 2011; 19: 1656-64.

[14] Voisin V, Caucheteur C, Mégret P, Albert J. Interrogation technique for TFBG-SPR refractometers based on differential orthogonal light states. Appl Opt 2011; 50: 4257-61.

[15] Caucheteur C, Chen C, Voisin V, Berini P, Albert J. A thin metal sheath lifts the EH to HE degeneracy in the cladding mode refractometric sensitivity of optical fiber sensors. Appl Phys Lett 2011; 99: 041118-1-3.

[16] Albert J, Shao LY, Caucheteur C. Tilted fiber Bragg grating sensors. Laser Photon Rev 2013; 7: 83-108.

[17] Iadicicco A, Cusano A, Cutolo A, Bernini R, Giordano M. Thinned fiber Bragg gratings as high sensitivity refractive index sensor. IEEE Photon Technol Lett 2004; 16: 1149-51.

[18] Caucheteur C, Bette S, Chen C, Wuilpart M, Mégret P, Albert J. Tilted fiber Bragg grating refractometer using polarizationdependent loss measurement. IEEE Photon Technol Lett 2008; 20: 2153-5.

[19] Caucheteur C, Wuilpart M, Chen C, Mégret P, Albert J. Quasidistributed refractometer using tilted Bragg gratings and time domain reflectometry. Opt Exp 2008; 16: 17882-90.

[20] Chen C, Albert J. Strain-optic coefficients of the individual cladding modes of a single mode fiber: theory and experiment. Electron Lett 2006; 42: 21-2
[21] White IM, Fan XD. On the performance quantification of resonant refractive index sensors. Opt Express 2008; 16: 1020-8.

[22] Chehura E, Murphy R, James S, Tatam R. Proceedings of the 18th International Conference on Optical Fiber Sensors; October 2006; SPIE, US 2006.

[23] Paladino D, Cusano A, Pilla P, Campopiano S, Caucheteur C, Mégret P. Spectral behavior in nano-coated tilted fiber Bragg gratings: effect of thickness and external refractive index. IEEE Photon Technol Lett 2004; 19: 2051-3.

[24] Lu YC, Geng R, Wang C, Zhang F, Liu C, Ning T, Jian S. Polarization effects in tilted fiber Bragg grating refractometers. J Lightw Technol 2010; 28: 1677-84.

[25] Renoirt JM, Zhang C, Debliquy M, Olivier MG, Mégret P, Caucheteur C. Proceedings of the SPIE Photonics Europe Conference; May 2012, SPIE, US 2012.

[26] Homola J. Surface plasmon resonance sensors for detection of chemical and biological species. Chem Rev 2008; 108: 462-93.

[27] Homola J, Ed. Surface Plasmon resonance based sensors. Springer series on chemical sensors and biosensors. Springer-Verlag, BerlinHeidelberg-New York 2006

[28] Alonso R, Subi as J, Pelayo J, Villuendas F, Tornos J. Singlemode, optical-fiber sensors and tunable wavelength filters based on the resonant excitation of metal-clad modes. Appl Opt 1994; 33: 5197-201.

[29] Diez A, Andres MV, Culverhouse DO, Birks TA. Cylindrical metal-coated optical fibre devices for filters and sensors. Electron Lett 1996; 32: 1390-2.

[30] Shevchenko Y, Francis TJ, Blair DAD, Walsh R, DeRosa MC, Albert J. In situ biosensing with a surface plasmon resonance fiber grating aptasensor. Anal Chem 2011; 83: 7027-34.

[31] Caucheteur C, Voisin V, Albert J. Polarized spectral combs probe optical fiber surface plasmons. Opt Express 2013; 21: 3055-3066.

[32] Sanders AW, Routenberg DA, Wiley BJ, Xia Y, Dufresne ER, Reed MA. Observation of plasmon propagation, redirection, and fan-out in silver nanowires. Nano Lett 2006; 6: 1822-1826.

[33] Bialiayeu A, Bottomley A, Prezgot D, Ianoul A, Albert J. Plasmonenhanced refractometry using silver nanowire coating on tilted fibre Bragg gratings. Nanotechnology 2012; 23: 44401

[34] Zhou W, Mandia DJ, Griffiths MBE, Bialiayeu A, Zhang Y, Gordon PG, Barry ST, Albert J. Polarization-dependent properties of the cladding modes of a single mode fiber covered with gold nanoparticles. Opt Exp 2013; 21:245-55.

[35] Shevchenko Y, Ahamad NY, Ianoul A, Albert J. In situ monitoring of the formation of nanoscale polyelectrolyte coatings on optical fibers using surface plasmon resonances. Opt Exp 2010; 18: 2040921.

[36] Shao LY, Coyle JP, Barry ST, Albert J. Anomalous permittivity and plasmon resonances of copper nanoparticle conformal coatings on optical fibers. Opt Mater Express 2011; 1: 128-37.

[37] Renoirt JM, Pilate J, Caucheteur C, Debliquy M, Olivier M, Damman P, Mégret P. Proceedings of the IEEE Photonics Society Benelux Chapter Symposium; December 2011; IEEE, US 2011.

[38] Caucheteur C, Mégret P, Cusano A. Tilted Bragg grating multipoint sensor based on wavelength-gated cladding-modes coupling. Appl Opt 2009; 48: 3915-20.

Received: March 25, 2013

Revised: March 25, 2013

Accepted: December 12, 2013

(C) Caucheteur et al.; Licensee Bentham Open.

This is an open access article licensed under the terms of the Creative Commons Attribution Non-Commercial License (http://creativecommons.org/licenses/by-nc/3.0/) which permits unrestricted, non-commercial use, distribution and reproduction in any medium, provided the work is properly cited. 